\title{
Automated 3-D Tissue Segmentation via Clustering
}

\author{
S. Edwards, S. Brown, M. Lee \\ Computational Sciences Division, U.S. Army Research Laboratory, Aberdeen Proving Ground, MD \\ michael.s.lee131.civ@mail.mil
}

\begin{abstract}
Generation of 3-D tissue models from medical imagery is useful for surgical planning and computer simulations, but often requires some amount of manual effort. In this work, we use the clustering algorithm, DBSCAN, in concert with a 3-D buildup procedure to automatically generate 3-D surface models of the brain and lungs from computed tomography head and chest scans, respectively. Extensions to other tissue types such as heart and liver are demonstrated for contrast-enhanced imagery.
\end{abstract}

Keywords: medical images, magnetic resonance imaging, computer-assisted tomography, clustering, DBSCAN

\section{Introduction}

Whole 3-D tissue segmentation is valuable for surgical planning and computer modeling and simulation. Specifically, the military and transportation sectors are interested in simulating the effects of various external trauma to hard and soft tissue [1]. Accurate models of organs from various individuals can improve the fidelity of these simulations. However, 3-D image segmentation can often involve a significant amount of tedious manual effort. We strive to automate this process for the convenient extraction of 3-D tissue models from stacked sets of computed tomography (CT) scans. While magnetic resonance imaging (MRI) may yield more detailed models, this modality is not suitable for individuals that may have metallic elements within their body, which could likely result from traumatic events.

Density-based spatial clustering of applications with noise [2], or DBSCAN, has become a popular tool for image segmentation due to its ability to 1 ) detect segments of arbitrary shape 2) and not require a priori specification of the number of segments to extract. These desirable features allow DBSCAN to be applied to a series of images with only determination of a single set of parameters. In the case of medical imagery, DBSCAN has been mostly used in the context of tumor segmentation. Remarkably versatile, DBSCAN can precisely segment lesions from images of skin [3] and tumors from a brain MRI [4]. Additionally, enhanced versions of DBSCAN properly extract lung nodules from CT scans [5].

Other algorithms have demonstrated promising results in medical image segmentation. The watershed algorithm [6] has been adapted to segmenting medical imagery. The algorithm can detect lung lesions from CT scans [7], much like the application of DBSCAN in this similar problem [5]. However, unlike the watershed approach, DBSCAN is more sensitive to the gray values of individual segments, allowing more 
S. Edwards, S. Brown, M. Lee; Automated 3-D Tissue Segmentation via Clustering. Journal of Biomedical Engineering and Medical Imaging, Volume 5, No 2, April (2018), pp 8-20

elaborate segmentation. Seeded region growth [8] is another popular method for medical image segmentation. There even exist fully automated versions of this algorithm specifically for medical imaging [9]. However, the method of region growing is limited to the segmentation of one tissue at a time. Since DBSCAN clusters the entire image, it is a more practical choice.

The construction of accurate patient-specific 3-D tissue models from medical scans has the potential to revolutionize surgical planning [10]; procedures already exist to optimize the 3-D printing of these models [11]. Historically, 3-D tissue segmentation is conducted manually [12] using tools such as 3DSlicer [13] and MIMICS 19.0 [14]. Recent advances in segmentation software have made semiautomatic approaches to segmenting medical images more accessible. However, there is a scarcity of fully automated procedures. The segmentation in these fully automated approaches is conducted either statistically or by simple thresholding depending on the desired tissue. For example, the liver is large in comparison to other organs in the abdomen and has a homogeneous intensity in medical images, allowing it to be extracted by statistically analyzing regions in the scan [15]. Furthermore, bone typically has a much higher intensity in medical imagery than other anatomical structures and can therefore be extracted by thresholding out other soft tissue [16]. The actual construction of the models can be handled through interpolation between stacked slices [16] or through minimizing a cost function [17].

In this work, we revisit the possibility of automated 3-D tissue segmentation. General realization of this goal for all tissue types would require human-level skill in the interpretation of medical imagery. Instead, we present a cluster-based segmentation algorithm that utilizes DBSCAN and a novel stacking algorithm to construct 3-D models of tissue.

\section{Theory}

\subsection{DBSCAN}

Unlike many other clustering algorithms, DBSCAN measures distance in terms of graph connectivity rather than a simple Euclidean metric, meaning that detected segments can deviate far from circularity. We briefly outline the method here. Define the $\varepsilon$-neighborhood of a point $p$ to be the set of points that are at most $\varepsilon$ from $p$. We call $p$ a core point if its $\varepsilon$-neighborhood contains at least $m$ points. A point $q$ is $\varepsilon$-reachable from $p$ if there exists a path of points $p_{1}=p, p_{2}, \ldots, p_{n}=q$ such that $p_{i}$ is a core point and its $\varepsilon$-neighborhood contains $p_{i+1}$ for all $1 \leq i \leq n-1$. A cluster is the maximal set of points $\varepsilon$-reachable from a core point $p$. Points that are not reachable from any core point are labeled as noise.

\subsection{Image Segmentation via DBSCAN}

Ye et al. [18] was one of the first groups to show that clustering algorithms, especially DBSCAN, can be used to segment images. Consider that each pixel $p_{i}$ of a CT scan image has coordinates $\left(x_{i}, y_{i}\right)$ and a gray value $c_{i}$. Given that we desire to cluster pixels by both spatial proximity and value, we treat each pixel as a 3-dimensional point, where the value becomes the third coordinate, ranging from 0 (black) to 255 (white). In DBSCAN terminology, a pixel, $p_{j}$, with coordinates $\left(x_{j}, y_{j}\right)$ and value $c_{j}$, is $\varepsilon$-reachable from $p_{i}$ if

$$
\left(x_{j}-x_{i}\right)^{2}+\left(y_{j}-y_{i}\right)^{2}+\alpha\left(c_{j}-c_{i}\right)^{2}<\epsilon,
$$


where the arbitrary scale factor $\alpha$ acknowledges that the dimensional units of location and value are incommensurate. For this work, we set $\alpha$ to 1 , because the height and width of typical CT scans are within an order of magnitude of the value range (256).

\subsection{3-D Voxel Segmentation}

Typically, 3-D tissue models can be derived by stacking 2-D medical scans [16]. For this work, we developed an algorithm to merge segments from 2-D DBSCAN segmentations resulting in multiple 3-D voxel segments, which are then converted into surfaces. The procedure begins with identifying unique segments $S_{a, 1}$ (where $a$ is the segment index) in the top scan in a given direction (e.g., axial). The second scan is then compared with the first, where each segment $S_{b, 2}$ is relabeled to account for matches with the first scan. When a segment $S_{b, 2}$ shares some of the same pixel locations with $S_{a, 1}$, and has at least $N$ corresponding pixel values that no more than $\tau$ apart in gray value, then that segment is relabeled to index of the segment from the first scan. If there is more than one common segment from the first scan for a given $S_{b, 2}$, the one with the most pixels in common "wins." If a segment $S_{b, 2}$ has no first scan counterparts, it is given a new index, incremented from all previous unique labels. This procedure is repeated for all scans, sequentially looping through adjacent scans. Then, the process is repeated starting backwards from the bottom scan, to merge segments that started off as two or more fragments from the top but, at some point, should have merged into one complete segment. With the series of 2 D segments now having been given common labels, they are merged into an individual voxel array per each unique label, and each voxel array is converted to a surface using the marching cubes algorithm [19]. For now, the generated surfaces must be manually selected for the wanted tissue type, but a fairly simple heuristic is that the larger surfaces tend to be the desired outputs.

\section{Methods}

\subsection{Databases}

Medical imagery, all in DICOM format, was downloaded from three separate databases. The first two databases were obtained from the Cancer Imaging Archive [20]. The Head-Neck Cetuximab study documents patients with stage III and IV head and neck cancer before and after treatment with the epidermal growth factor receptor (EGFR) inhibitor Cetuximab [21]. From this repository, we obtained three full body series that were utilized for brain extraction. The second database from this archive, named LungCT-Diagnosis, contained series of scans with a slice thickness between three to six millimeters that were taken for diagnosis prior to surgery [22]. These images were enhanced with a contrast medium, allowing the heart and liver to be segmented in addition to the lungs. The final database was the result of a collaboration between the ELCAP and VIA research groups to create a dataset for detection software [23]. It consists of series of unenhanced chest CT scans possessing a slice thickness of $1.25 \mathrm{~mm}$. Without the contrast medium, only the lungs were extracted from this last database. We leave it as future work to determine the process to extract 3-D models of the heart and liver from unenhanced scans. 
S. Edwards, S. Brown, M. Lee; Automated 3-D Tissue Segmentation via Clustering. Journal of Biomedical Engineering and Medical Imaging, Volume 5, No 2, April (2018), pp 8-20

\subsection{Procedure}

\subsubsection{Preprocessing}

The DICOM files are first read into our program using VTK tools [24]. CT scans reflect the radiodensity of tissue measured in Hounsfield units (HU) [25]. Air and water at standard temperature and pressure have defined radiodensity values of -1000 and $0 \mathrm{HU}$ respectively. A preliminary thresholding is applied to highlight certain desired tissue and therefore refine the segmentation process. The image is then converted from HU to grayscale by simply scaling the data. To remove extraneous noise while retaining edge integrity, two passes of bilateral filtering [26] are applied to the images.

\subsubsection{Segmentation}

Before clustering in bulk, we first establish the values for DBSCAN parameters to best extract different tissue types. We define the minimum number of points needed in the declaration of a core point $m$ to be some fraction $f$ of the area of the $\varepsilon$-neighborhood - that is,

$$
m=f \pi \epsilon^{2} .
$$

The circular assumption of nearby points can be used since the pixels of an image are, by definition, densely packed on a square lattice. With fractions $f<1$, regions with sharp, non-circular edges can be detected. Experimentation with $\varepsilon, f$, and thresholding values was conducted to determine sufficient values for each tissue type, as seen in Table 1.

Table 1: The values of $\varepsilon$, the fraction of the area of the $\varepsilon$-neighborhood $f$, and the thresholding values in $H U$ that were determined to be suitable for DBSCAN-based segmentation of each tissue type.

\begin{tabular}{|c|c|c|c|c|}
\hline Tissue Type & $\boldsymbol{\varepsilon}$ & $\boldsymbol{f}$ & Min & Max \\
\hline Brain & 4 & 0.1 & -50 & 200 \\
\hline Lung & 8 & 0.3 & -800 & -300 \\
\hline Heart (contrasted) & 4 & 0.2 & -50 & 200 \\
\hline Liver (contrasted) & 6 & 0.4 & -50 & 200 \\
\hline
\end{tabular}

\subsubsection{3-D Construction}

The buildup portion of our algorithm requires two additional parameters when determining whether to merge two segments from different slices: the number $N$ of corresponding pixel locations between two segments whose values must be no more than $\tau$ apart. Through experimentation, we determined the values of $N=50$ and $\tau=20$ produced a clean result with the high resolving power of smaller structures.

\subsection{Software}

All the algorithms for this work were written in Python, using various common add-on modules. Scikitimage was used to process images and Scikit-learn was used to perform DBSCAN clustering [27]. Besides initial DICOM reading, the VTK toolkit [24] was also used to create the 3-D models. The standardformat triangulated-surface STL files were rendered in Paraview [28]; the inclusion of OSPray [29] enhanced surface display by adding ambient occlusion and shadows. 


\section{Results and Discussion}

\subsection{The 2-D Case}

We experimented with different clustering algorithms that did not require the number of clusters to be specified as a parameter. The watershed method simply outlines regions, impeding the reliance of the buildup algorithm on merging filled segments together; therefore, it was disregarded. We also ruled out the region growing method because of its inability to simultaneously segment more than one region from the image. Ultimately, we narrowed our search to DBSCAN and mean shift, an algorithm that computes clusters by finding modes of the underlying probability density function of the data [30].

1)

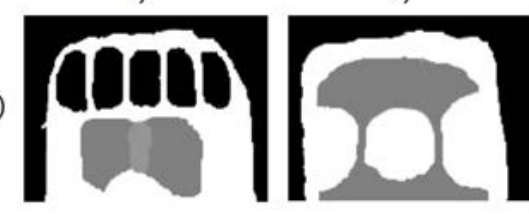

b)
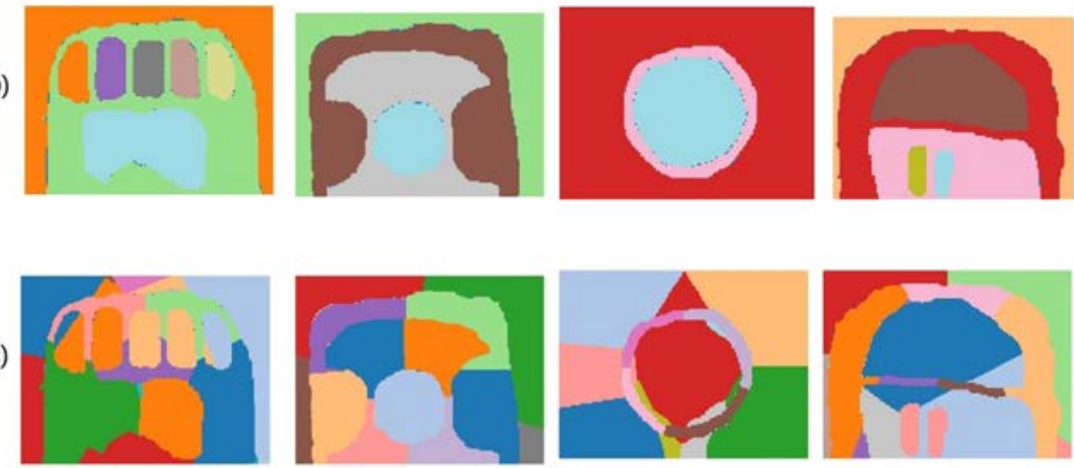

Figure 1: The toy images (a) and the results of applying DBSCAN (b) with $\varepsilon=4$ and $f=0.2$ and mean shift (c).

By constructing toy models that mimicked various features of the skull, such as a thin border between two similarly colored regions, we determined that DBSCAN would operate more effectively on medical images. Notice that for every case in Figure 1, mean shift creates more clusters than necessary by segmenting large regions of homogeneous color, a disconcerting feature for a segmentation algorithm. On the other hand, DBSCAN accurately segments each image, even ignoring the abundance of noise present in image (a3). 
a)

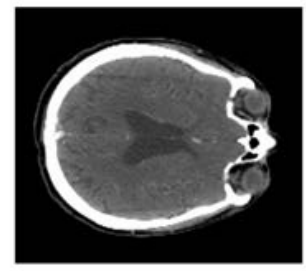

b)

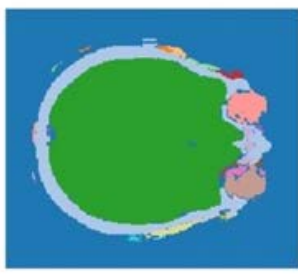

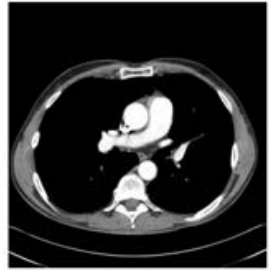

c)

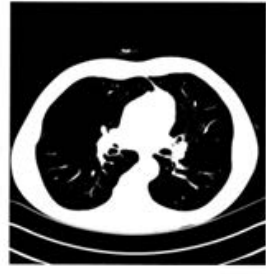

d)

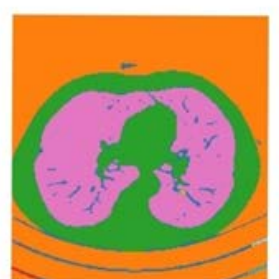

e)

Figure 2: A CT scan of a human skull (a) and chest cavity (c) and the resulting segmentation by DBSCAN (b) and (e) respectively, using the parameters from Table 1 . The thresholded chest cavity (d) is included to emphasize the bronchial tubes that are masked in the original image.

\subsection{3-D Models}

\subsubsection{Bone}

Segmenting bone from CT scans may be done trivially through simple thresholding as its values are typically higher than that of surrounding tissue. However, utilizing the same parameters as the brain $(\varepsilon$ $=4$ and $f=0.1$ ), our algorithm can provide a more precise segmentation of bone than that from thresholding.

Thresholding is less effective for several reasons. Foreign objects such as metals register very highly on the Hounsfield scale; therefore, they are captured by the thresholding, as evidenced by the inclusion of the bed frame in row (b) of Figure 3. Additionally, various bone types yield different Hounsfield values. For example, the skull bones have a much higher value than that of the ribs. Notice in row (b) of Figure 3 that the skull is well-defined, but the clavicle, ribs, and sternum are severely lacking. Although our algorithm separates the skeleton into three clusters in this case, it provides much more detail globally and does not include any extraneous materials, such as the metal bed frame. 
a)
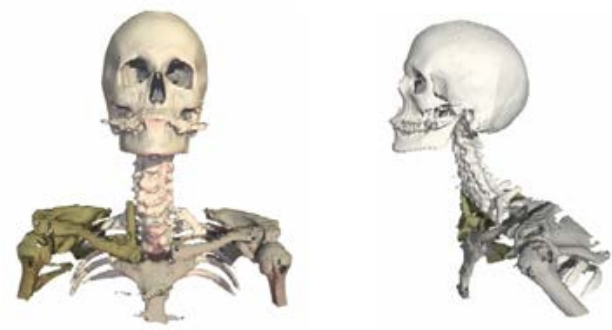

b)
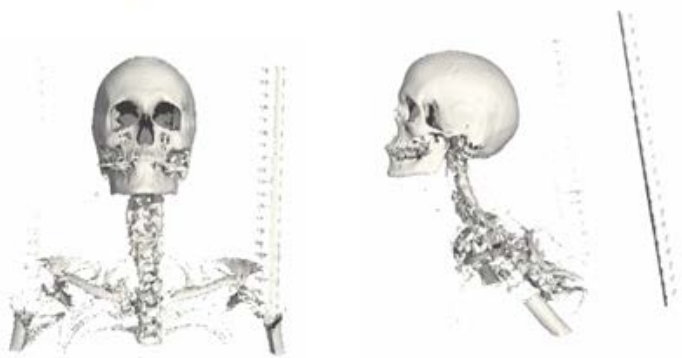

Figure 3: The results of segmentation of the skeleton of patient 0013 of the Head-Neck Cetuximab study [21] by our algorithm (a) and thresholding (b) respectively. Our algorithm segmented the skeleton into three separate regions, reflected by the different colors.

\subsubsection{Brain}

Segmenting the brain yields some of the most promising and repeatable results from this algorithm, likely because the brain is surrounded by the skull, providing a clear separation from other soft tissue. It is even possible to cluster the brain and spinal cord together, as evidenced by Figure 4. However, this feature does not appear in brain models segmented from full body scans. The intricate creasing present on the back of the brain and the detailed cerebellum exemplify the power of this algorithm. The folding pattern of the brain is unable to be detected both because of the loss of detail in the CT scans and the necessary application of bilateral filtering [24].
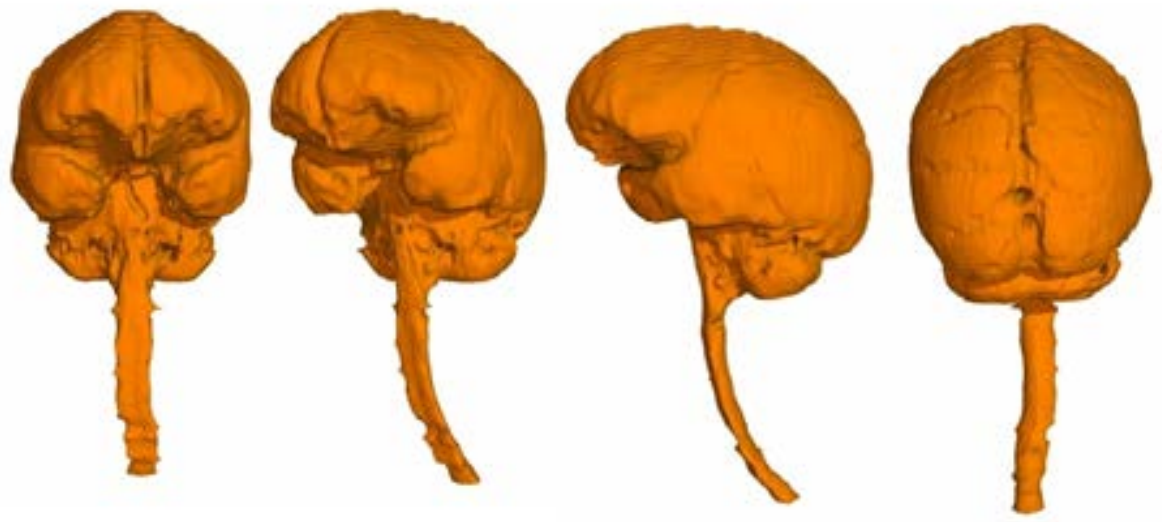

Figure 4: The segmented brain from patient 0013 from the Head-Neck Cetuximab study [21]. 
S. Edwards, S. Brown, M. Lee; Automated 3-D Tissue Segmentation via Clustering. Journal of Biomedical Engineering and Medical Imaging, Volume 5, No 2, April (2018), pp 8-20

The brain models appearing in Figure 5 are taken from full body scans. The lack of attachment to the spinal cord is likely because the spinal cord is typically clustered with back muscles in lower torso scans or sometimes missed altogether. While the parameters for the segmentation are more attuned to the brain segmented in Figure 4, the fact that detailed brain models can be extracted from other patients demonstrates the reproducibility of the algorithm.

a)

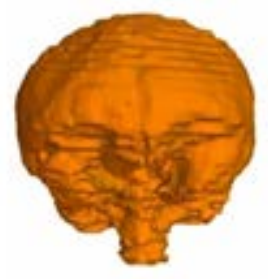

b)

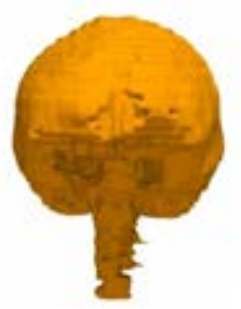

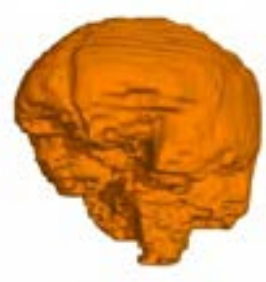

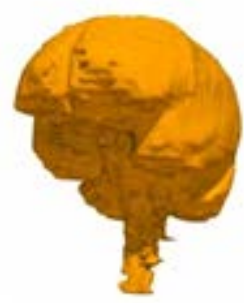

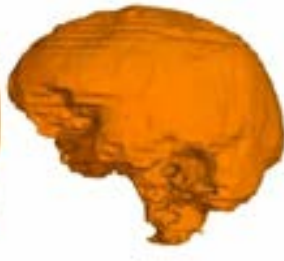
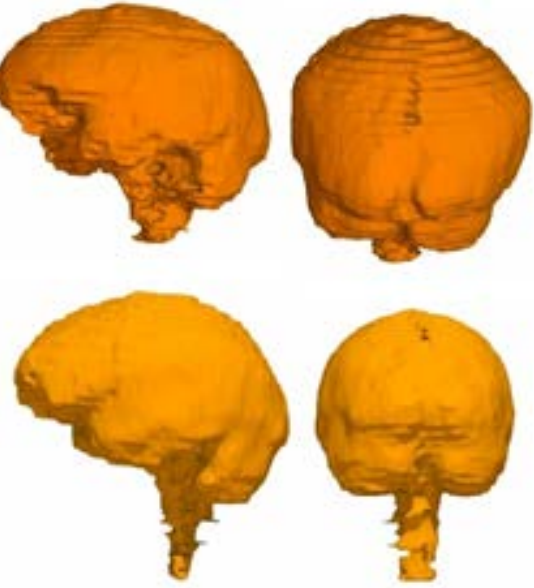

Figure 5: The segmented brains from patient 0001 (a) and 0009 (b) from the Head-Neck Cetuximab study [21].

\subsubsection{Lungs}

The lungs stand out in stark contrast with the rest of the soft tissue in the body because of the air contained within them. There is a simple way to segment the lungs by extracting their imprint; however, this method is impractical, as it ignores the bronchial tubes. The ideal lung segmentation should include some details of the bronchial tubes and a clear majority of the lung tissue.
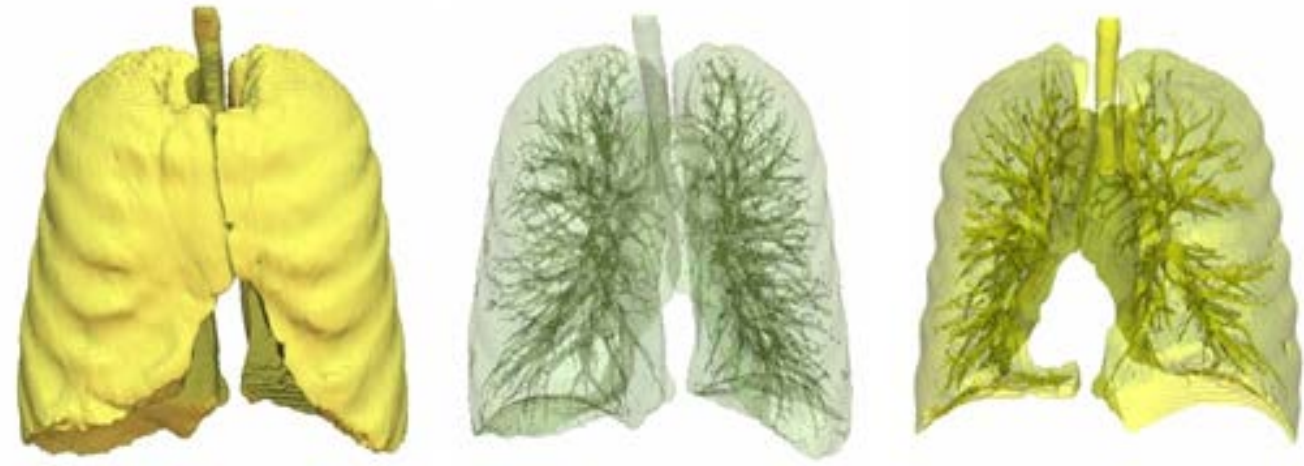

Figure 6: Segmentation results of the lungs of patient R0006 of the LungsCT-Diagonsis study [22].

Our protocol can not only depict the shape of the outer lung but discern a great amount of detail from the bronchial tubes as well, as illustrated by Figure 6 . This trend seems to be reproducible as well. When applied to all fifty lungs in the ELCAP-VIA database, our algorithm performed quite well by accurately segmenting most of the lungs; this data is included in the supplementary materials. 


\subsubsection{Other Tissue}

Our protocol has shown promising results for extracting other tissue types. It could segment the heart and liver in scans enhanced with contrast medium as seen in Figs. 7 and 8. However, the algorithm failed when the same parameters were applied to uncontrasted scans. In the uncontrasted case, the gray values of the heart closely resemble those of the chest muscles; similarly, it is difficult to discern the liver from the surrounding abdominal tissue.
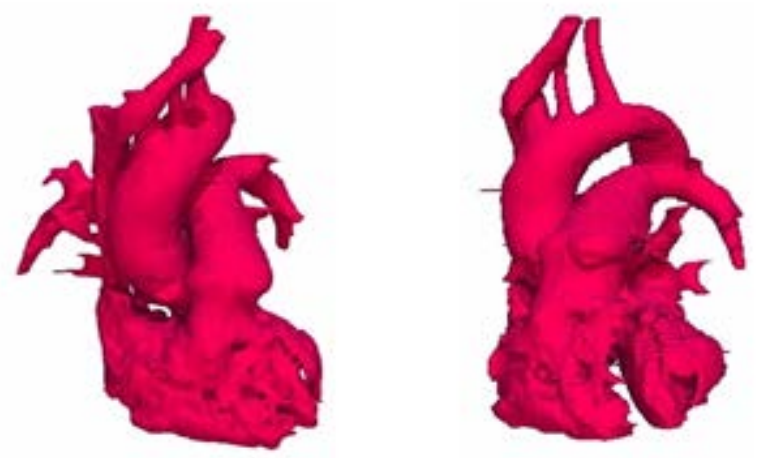

Figure 7: Segmentation results of a contrasted heart of patient R0006 of the LungsCT-Diagonsis study [22].

The segmentation displays a detailed contouring of the aorta and pulmonary artery. Even the superior vena cava is intricately defined. However, apparent gaps exist on the body of the heart and no chamber information can be discerned when the surface is made partially transparent.
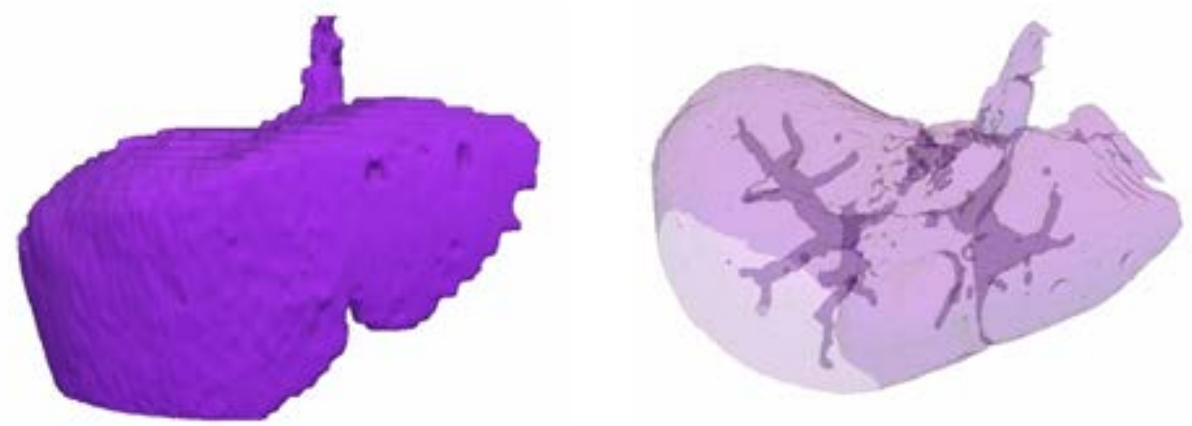

Figure 8: Segmentation results of a contrasted liver of patient R0006 of the LungsCT-Diagonsis study [22].

Our approach is able to properly capture some extent of the hepatic ducts, although it was unable to detect the ligament that splits the liver.

Segmenting muscle tissue has proven to be particularly challenging. The segments are typically in many pieces and lacking large chunks of tissue. One likely explanation is the variability of muscle shape throughout the body is not conducive for a global set of parameters. Additionally, muscle tissue is very similar in appearance to other soft tissue in the body which could disrupt segmentation. 
S. Edwards, S. Brown, M. Lee; Automated 3-D Tissue Segmentation via Clustering. Journal of Biomedical Engineering and Medical Imaging, Volume 5, No 2, April (2018), pp 8-20

\subsection{Limitations}

Although our procedure provides a convenient and efficient method for 3-D segmentation, it is by no means a perfect solution to the problem. Certain unavoidable phenomenon in the scans themselves could result in undesirable outcomes. The CT scans often yield a circular working image; a black background fills the remainder of the square frame. In off-center scans, the lungs may be clustered with this black background by DBSCAN, as in Figure 9. However, this problem may be remedied by either cropping the image so that only the circular scan is processed or by setting the black background to white.

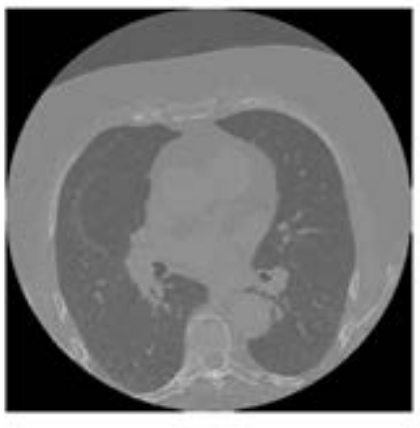

a)

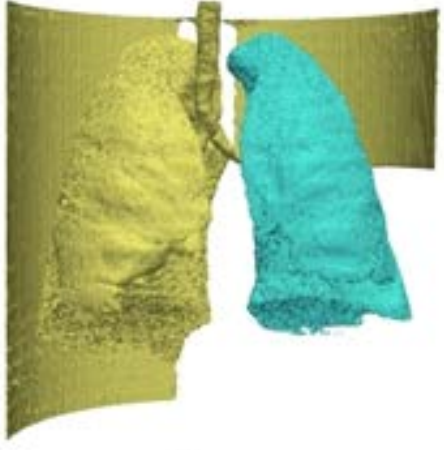

b)

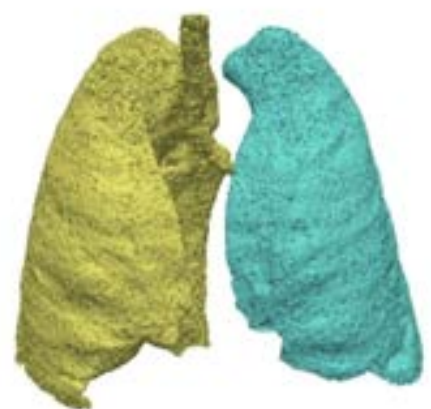

c)

Figure 9: Depicting the impact of off-center CT scans from patient W0008 in the ELCAP-VIA database [23]. The sample scan (a) and the results of the segmentation algorithm if run normally (b). By setting the outer region of the scan from black to white, the segmentation results were much more accurate (c). The colors in (b) and (c) represent the different segments detected.

Another obstruction to clean segmentation is the presence of concentrated dyes or foreign objects with a high radiodensity such as metals. This creates a bright spot that extends outwards in a gradient, disrupting our algorithm. Since we view color as a third dimension, the gradient creates a ramp, possibly causing points from two separate regions to be $\varepsilon$-reachable. In the case of patient W0014 depicted in Figure 10, the lungs in this slice were merged with the background, creating a region that exceeded our threshold of pixel size for segments. Therefore, the top quarter of the left lung was unable to be detected by our algorithm. If we had decreased $\varepsilon$ to incorporate this region, then the entire background would have been included in the segmentation, masking the lungs.

a)

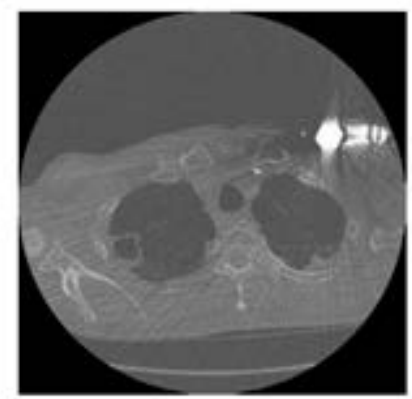

b)

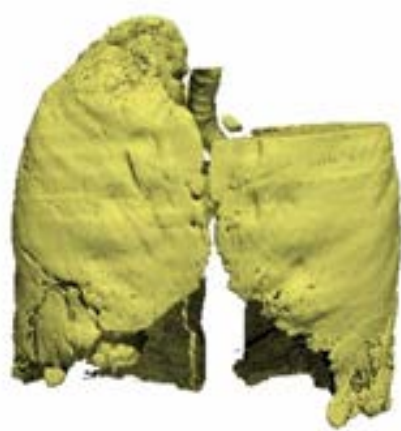

Figure 10: The sample scan (a) and the results of the segmentation algorithm (b) that depict the impact of a bright spot from patient W0014 in the ELCAP-VIA database [23]. 
Finally, widespread noise can be detrimental to the segmentation, as seen acutely for the detailed extraction of bronchial tubes. While an abundance of noise seems to be almost negligible for the segmentation of soft tissue of homogeneous density like the brain and liver, it can profoundly impact the segmentation of lung tissue.

a)

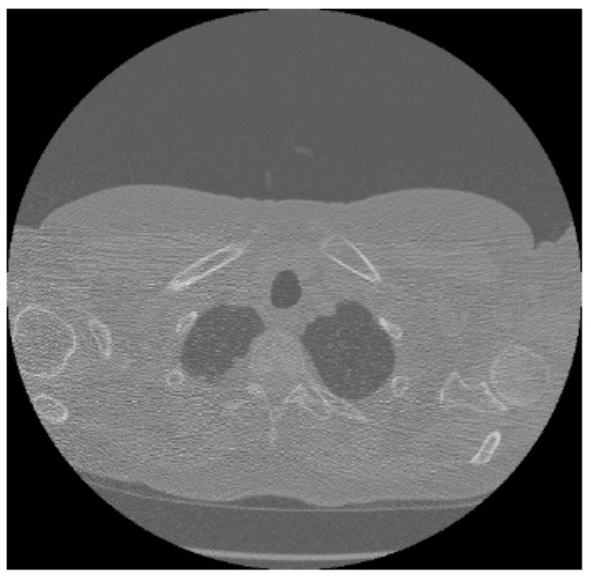

b)

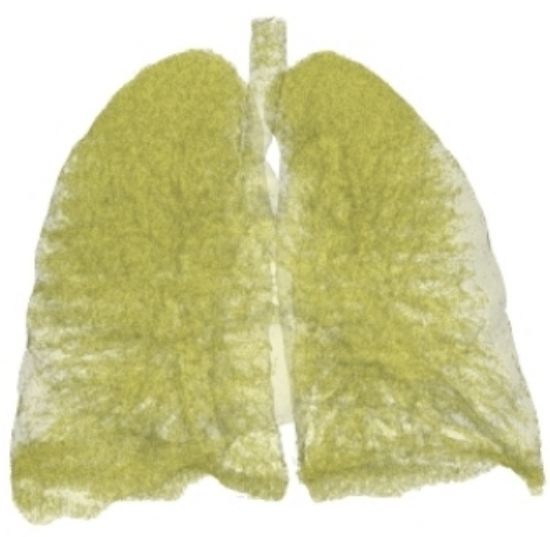

Figure 11: The results of a noisy scan on patient W0007 in the ELCAP-VIA database [23]. The sample scan (a) and the results of the segmentation algorithm (b).

Notice the white speckles present over the lung tissue in image (a) of Figure 11. DBSCAN will not cluster this noise with the lung tissue; therefore, very little detail from the bronchial tubes can be detected. Furthermore, intense noise can cause the inside of the lungs to appear spongy, which not only masks the inside of the lungs but could lead to a misdiagnosis as well, if these models were used in a clinical context.

\section{Conclusion}

Automated 3-D segmentation of various tissue types could impact surgical planning, medical training, diagnosing patients, and simulation from real tissue models. Although a robust universal solution will likely require more sophisticated approaches, we have developed a simple-to-use protocol for fully automated 3-D segmentation of certain tissue types. This algorithm does possess a few limitations, but its ability to accurately segment multiple organs demonstrates its versatility. We present a method to extract the heart and liver from scans enhanced with contrast medium; extending the results to unenhanced scans is reserved for future work. Furthermore, extending this work to MRI may yield more detailed results for certain tissue types where CT scans have limited resolving power.

\section{REFERENCES}

[1]. A. N. Ranslow, et al., Microstructural analysis of porcine skull bone subjected to impact loading. ASME 2015 International Mechanical Engineering Congress and Exposition, American Society of Mechanical Engineers, 2015. 
S. Edwards, S. Brown, M. Lee; Automated 3-D Tissue Segmentation via Clustering. Journal of Biomedical Engineering and Medical Imaging, Volume 5, No 2, April (2018), pp 8-20

[2]. M. Ester, et al., A density-based algorithm for discovering clusters in large spatial databases with noise. Kdd, Vol. 96, 1996, pp. 226-231.

[3]. M. E. Celebi, Y. A. Aslandogan, P. R. Bergstresser, Mining biomedical images with density-based clustering. Information Technology: Coding and Computing, 2005. ITCC 2005. International Conference on, Vol. 1, IEEE, 2005, pp. 163-168.

[4]. S. K. Bandyopadhyay, T. U. Paul, Segmentation of brain tumour from mri image analysis of k-means and dbscan clustering. International Journal of Research in Engineering and Science 1 (1) (2013) 48-57.

[5]. W. Zhang, X. Zhang, J. Zhao, Y. Qiang, Q. Tian, X. Tang, A segmentation method for lung nodule image sequences based on superpixels and density-based spatial clustering of applications with noise. PloS one $12(9)$.

[6]. L. Vincent, P. Soille, Watersheds in digital spaces: an efficient algorithm based on immersion simulations. IEEE Transactions on Pattern Analysis \& Machine Intelligence (6) (1991) 583-598.

[7]. Y. Tan, L. H. Schwartz, B. Zhao, Segmentation of lung lesions on ct scans using watershed, active contours, and markov random field. Medical physics 40 (4).

[8]. R. Adams, L. Bischof, Seeded region growing. IEEE Transactions on pattern analysis and machine intelligence 16 (6) (1994) 641-647.

[9]. R. Pohle, K. D. Toennies, Segmentation of medical images using adaptive region growing. Proc. SPIE Medical Imaging, Vol. 4322, 2001, pp. 1337-1346.

[10]. N. J. Mankovich, D. Samson, W. Pratt, D. Lew, J. Beumer 3rd, Surgical planning using three-dimensional imaging and computer modeling. Otolaryngologic Clinics of North America 27 (5) (1994) 875-889.

[11]. T. M. Bücking, E. R. Hill, J. L. Robertson, E. Maneas, A. A. Plumb, D. I. Nikitichev, From medical imaging data to $3 d$ printed anatomical models. PloS one 12 (5).

[12]. K. H. Höhne, W. A. Hanson, Interactive 3d segmentation of mri and ct volumes using morphological operations. Journal of computer assisted tomography 16 (2) (1992) 285-294.

[13]. A. Fedorov, et al., 3d slicer as an image computing platform for the quantitative imaging network. Magnetic resonance imaging 30 (9) (2012) 1323-1341.

[14]. Materialise, Mimics software, http://www.materialise.com (2016).

[15]. L. Massoptier, S. Casciaro, A new fully automatic and robust algorithm for fast segmentation of liver tissue and tumors from ct scans. European radiology 18 (8) (2008) 1658.

[16]. K. Subburaj, B. Ravi, High resolution medical models and geometric reasoning starting from ct/mri images. 10th IEEE International Conference on Computer-Aided Design and Computer Graphics, IEEE, 2007, pp. 441-444. 
[17]. T. Heimann, I. Wolf, H.-P. Meinzer, Active shape models for a fully automated $3 d$ segmentation of the liver-an evaluation on clinical data. Medical Image Computing and Computer-Assisted InterventionMICCAI 2006 (2006) 41-48.

[18]. Q. Ye, W. Gao, W. Zeng, Color image segmentation using density-based clustering. Multimedia and Expo, 2003. ICME'03. Proceedings. 2003 International Conference on, Vol. 2, IEEE, 2003, pp. II-401.

[19]. W. E. Lorensen, H. E. Cline, Marching cubes: A high resolution 3d surface construction algorithm. ACM siggraph computer graphics, Vol. 21, ACM, 1987, pp. 163-169.

[20]. K. Clark, et al., The cancer imaging archive (TCIA): maintaining and operating a public information repository. Journal of digital imaging 26 (6) (2013) 1045-1057.

[21]. W. R. Bosch, W. L. Straube, J. W. Matthews, J. A. Purdy, Data from head-neck cetuximab. Tech. rep., The Cancer Imaging Archive (2015).

[22]. O. Grove, et al., Quantitative computed tomographic descriptors associate tumor shape complexity and intratumor heterogeneity with prognosis in lung adenocarcinoma. PloS one 10 (3 (2015) e0118261.

[23]. A. P. Reeves, Y. Xie, S. Liu, Large-scale image region documentation for fully automated image biomarker algorithm development and evaluation. Journal of Medical Imaging 4 (2) (2017) 024505-024505.

[24]. W. J. Schroeder, B. Lorensen, K. Martin, The visualization toolkit: an object-oriented approach to 3D graphics, Kitware, 2004.

[25]. G. N. Hounsfield, Computerized transverse axial scanning (tomography): Part 1. Description of system. The British journal of radiology 46 (552) (1973) 1016-1022.

[26]. C. Tomasi, R. Manduchi, Bilateral filtering for gray and color images. Computer Vision, 1998. Sixth International Conference on, IEEE, 1998, pp. 839-846.

[27]. E. Jones, et al., SciPy: Open source scientific tools for Python (2001). URL http://www.scipy.org/

[28]. J. Ahrens, B. Geveci, C. Law, Paraview: An end-user tool for large data visualization, The Visualization Handbook 717.

[29]. I. Wald, G. P. Johnson, J. Amstutz, C. Brownlee, A. Knoll, J. Jeffers, J. Günther, P. Navratil, Ospray-a cpu ray tracing framework for scientific visualization. IEEE transactions on visualization and computer graphics 23 (1) (2017) 931-940.

[30]. D. Comaniciu, P. Meer, Mean shift: A robust approach toward feature space analysis. IEEE Transactions on pattern analysis and machine intelligence 24 (5) (2002) 603-619. 